\title{
Corela
}

Cognition, représentation, langage

HS-25 | 2018

Les procédés implicites dans l'interface sémantiquepragmatique

\section{Sens implicite, implicatures et principes d'inférence}

\author{
Olivier SIMONIN
}

\section{CpenEdition}

Journals

Édition électronique

URL : http://journals.openedition.org/corela/6511

DOI : $10.4000 /$ corela.6511

ISSN : 1638-573X

Éditeur

Cercle linguistique du Centre et de l'Ouest - CerLICO

Référence électronique

Olivier SIMONIN, « Sens implicite, implicatures et principes d'inférence », Corela [En ligne],

HS-25 | 2018, mis en ligne le 12 juillet 2018, consulté le 19 avril 2019. URL : http://

journals.openedition.org/corela/6511; DOI : 10.4000/corela.6511

Ce document a été généré automatiquement le 19 avril 2019

\section{cc) (†) (9)}

Corela - cognition, représentation, langage est mis à disposition selon les termes de la licence

Creative Commons Attribution - Pas d'Utilisation Commerciale - Partage dans les Mêmes Conditions

4.0 International. 


\title{
Sens implicite, implicatures et principes d'inférence
}

\author{
Olivier SIMONIN
}

\section{Introduction}

1 L'objectif de cette contribution est de proposer l'ébauche d'une conception de l'implicite en plaçant les questions du rôle des principes d'inférence et de la dérivation du sens implicite au premier plan. Cette réflexion n'entend évidemment pas épuiser la question de l'implicite, mais plutôt explorer plusieurs pistes et hypothèses liées à sa reconstruction. Nous allons tout d'abord tenter de caractériser ce qu'est l'implicite, par contraste avec ce qui relève de l'explicite dans la communication verbale. Le problème de la délimitation entre contenus implicites et contenus explicites se révèle être plus problématique qu'il n'apparaît de prime abord. Nous nous interrogerons ensuite sur les principes d'inférence postulés par Grice et les théories pragmatiques post-gricéennes (principe de coopération, principe de pertinence), puis sur leur implication précise dans la dérivation du sens. Nous contrasterons à cette fin le modèle parallèle de Recanati (2004) et celui de Sperber et Wilson (1995 [1986], 2004, 2012), pour suggérer quelque nouvelles pistes concernant l'implicite et la reconstruction du sens.

\section{L'explicite et le sens implicite}

2 La catégorie de l'implicite est difficile à circonscrire car des inférences, traditionnellement associées à l'implicite, se révèlent nécessaires afin de définir les propositions logiques qui correspondent au premier sens des énoncés. Ce sens se trouve le plus souvent sous-déterminé par les formes linguistiques employées. Un des apports de la pragmatique post-gricéenne a été de montrer que la dichotomie opérée entre explicite et implicite n'est pas aussi nette qu'on a été porté à le croire jusqu'à Grice. 


\subsection{Contenus implicites, contenus explicites}

3 Kerbrat-Orecchioni $(1998: 20)$ contraste contenus explicites et contenus implicites, à la suite de Grice dont elle reprend les travaux. Les contenus explicites équivalent à ce qui est posé, «ce qui est dit » en termes gricéens - disons, en première analyse, au sens propositionnel correspondant aux énoncés - alors que les contenus implicites ne sont pas posés explicitement mais inférés voire imposés (étant donnés comme pouvant être inférés ou bien comme déjà connus); ils concernent ce qui est véhiculé en surplus, par implication. Voici comment nous schématisons l'opposition, telle qu'elle est présentée par Kerbrat-Orecchioni (qui distingue immédiatement, parmi les contenus implicites, présuppositions et sous-entendus - opposition classique reprise sous les termes qu'utilise Ducrot dès 1969, qu'il ne nous paraît pas pertinent de développer ici) :

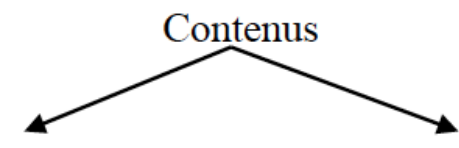
Explicites
Implicites
(ce qui est dit, posé)
(ce qui est inféré/impliqué, voire imposé)

Suivant cette conception, le sens implicite est défini en creux, par opposition au sens explicite. La conception selon laquelle les contenus explicites sont toujours posés et que les contenus implicites sont toujours inférés voire imposés ${ }^{1}$ est démentie par le fait que certains contenus peuvent être imposés tout en étant codés, c'est-à-dire donnés de manière tout à fait explicite :

(1) P. - (...) vous ne voulez pas vous rendre compte que le résultat aussi déplorable qu'il soit n'est rien d'autre qu'un résultat normal face à une politique aveugle - et je veux absolument je veux...

C. - C'est vous qui dites que c'est une politique aveugle. (Moeschler 1981:60, tel que cité

par Kerbrat-Orecchioni 1998 : 33)

La politique en question est explicitement décrite comme aveugle, et le locuteur P cherche à imposer l'idée qu'il s'agit d'une politique aveugle en nommant explicitement le référent une politique aveugle. Son allocutaire, $\mathrm{C}$, refuse cette idée en revenant sur la désignation choisie. Bien qu'il y ait ici une imposition effectuée au niveau du sens, refusée par $\mathrm{P}$, le contenu véhiculé reflète bien le contenu purement sémantique des formes linguistiques employées. Il est donc inapproprié de parler de contenu implicite, même si le contenu explicite du syntagme une politique aveugle, posé dans le cadre d'une assertion, ne fait pas l'objet d'une assertion séparée (que les énoncés Cette politique est aveugle ou C'est une politique aveugle, mettons, effectueraient). Il est ainsi essentiel de définir la distinction autrement, sans retenir le critère d'imposition pour déterminant.

\section{2. «Ce qui est dit » et les implicatures}

En suivant Grice et, notamment, sa (re)lecture par Recanati (2004), nous définirons « ce qui est dit » comme la proposition à laquelle correspond un énoncé dans la situation où celui-ci est produit. L'énoncé (2)

(2) J'ai bien bu à la soirée d'hier. 
véhicule explicitement une proposition que l'on pourrait gloser ainsi :

Au moment $t$ où le locuteur de (2) s'exprime, ce dernier a bu beaucoup d'alcool à la

soirée qui s'est déroulée le jour précédant celui où se situe $t$.

De plus, en fonction du contexte, (2) peut signifier bien autre chose en surplus de son sens explicite :

(2a) Je n'aurais pas dû boire tant.

(2b) Je n'ai plus envie de boire de l'alcool.

(2c) Je n'accepte pas ton invitation à le faire.

9 Ces sens, qui peuvent très bien se cumuler, constituent potentiellement l'essentiel $d u$ message signifié par l'énoncé. Ils ne relèvent pas de «ce qui est dit» mais ils correspondent à des implicatures qui, pour Récanati (2004: 17), sont générées par des processus pragmatiques secondaires déclenchés par l'application du principe de coopération décliné en maximes, dans une perspective gricéenne. Or qui dit processus pragmatiques secondaires entend qu'il existe des processus pragmatiques primaires. Que sont ces processus pragmatiques primaires, quelle est leur nature?

Les processus primaires permettent de déterminer "ce qui est dit », d'attribuer un sens propositionnel à l'énoncé proféré, de reconstruire à partir de celui-ci une proposition logique complète et interprétable. A cette fin, les pragmaticiens post-gricéens reconnaissent plusieurs mécanismes que l'on peut regrouper en quatre grandes catégories : ${ }^{2}$

(i) la désambiguïsation : attribution d'un sens unique à des syntagmes ambigus

(ii) la référenciation : attribution d'un référent à tout syntagme référentiel

(iii) le comblage/ la saturation : remplissage d'une place logique laissée vide

(iv) la modulation : adaptation, modification du sens d'un syntagme

11 Nous illustrerons ces catégories en montrant leur implication dans la reconstruction du sens propositionnel que l'on associe naturellement à (2) :

2i. soirée renvoie à une fête et non au soir en soi.

2ii. Je renvoie au locuteur, qui est le référent visé.

2iii. Ce qui a été bu est une boisson (quand on boit, on boit quelque chose, la relation dite par boire implique nécessairement un buveur et un contenu bu).

2iv. Ce qui a été bu était alcoolisé (ce que ne dit pas, à proprement parler, le sens littéral).

On peut ainsi reconstruire la proposition à laquelle correspond un énoncé donné. Il convient toutefois de noter que cette proposition est souvent modulée voire enrichie. Elle n'est alors pas minimale, reflétant un sens littéral dans une acception étroite du terme, et dépasse le strict sens propositionnel que l'on attribue directement aux énoncés en logique pure. Ce choix se justifie, dans une perspective post-gricéenne, du fait que les allocutaires interprètent le sens propositionnel des énoncés tout en effectuant automatiquement les ajustements que couvre la modulation. ${ }^{3}$ Parallèlement à la dérivation de ce sens propositionnel (déjà potentiellement modulé), l'allocutaire infère s'il y a lieu des implicatures qui viennent s'ajouter à ce sens.

$13 \mathrm{Au}$ vu de l'importance des procédés nécessaires pour déterminer le sens propositionnel auquel correspondent les énoncés, il faut fréquemment un travail d'inférence important ne serait-ce que pour déterminer ce qui est dit, et nous souscrivons pleinement à l'observation de Noveck et Sperber (2007: 189), pour qui «les formes linguistiques ne servent pas à coder le sens véhiculé par le locuteur, mais à l'indiquer $"{ }^{4}$ et, plus généralement, à l'idée que ces formes sous-déterminent le sens, ce qui est désormais 
quasiment un lieu commun en pragmatique. Partant, nous définirons le contenu explicite d'un énoncé comme le squelette linguistique et sémantique à partir duquel se reconstruit ce qui est dit. Suivant une suggestion de Sperber et Wilson $(1995: 182,2012: 13)$, on peut proposer que le caractère explicite d'un énoncé est une affaire de degrés, et que plus grand est le rôle joué par le code linguistique dans l'interprétation d'un énoncé, plus explicite sera l'énoncé en question. De cela il découle que définir l'implicite par rapport à l'explicite paraît une entreprise pour le moins hasardeuse, étant donné qu'un certain nombre d'inférences - et d'une dose implicite par conséquent - est déjà nécessaire pour déterminer « ce qui est dit ».

\subsection{Modulation, tropes et implicite}

14 Suivant la conception du sens que nous venons d'esquisser, les métaphores et les autres tropes se rattachent à la modulation et à la reconstruction de «ce qui est dit ». Cela va à l'encontre de Grice $(1975,1989)$, qui donne - entre autres - pour exemple le recours à la métaphore comme une violation de la première maxime de qualité ( Ne dites pas ce que vous croyez être faux ", $1989: 27)$ générant une implicature, venant effacer et remplacer le sens initial (You are the cream of my coffee, "Tu es la crème de mon café » pouvant s'interpréter comme 'Tu es ma fierté et ma joie', 1989: 34). Cependant, à partir du postulat que pour déterminer "ce qui est dit» il est d'abord nécessaire de fixer la référence des syntagmes qui en appellent une; il devient alors nécessaire d'inclure la métaphore (et les tropes) au sein de ce niveau: il n'est en effet pas possible de reconstruire la proposition logique correspondant à l'énoncé Le loup de Wall Street, c'est John Belfort sans comprendre que ce loup est un loup métaphorique.

(3) Le loup de Wall Street, c'est John Belfort.

En d'autres termes, pour pouvoir attribuer une valeur de vérité à la proposition qui correspond à (3), il convient d'abord de reconstruire celle-ci, et cela demande d'avoir immédiatement accès au sens figuratif, métaphorique de l'expression le loup de Wall Street. De la même manière, dans cet exemple emprunté à Recanati (2004: 63), la métonymie doit être élucidée pour reconstruire "ce qui est dit ", sachant qu'il est question d'un guerrier dont le bouclier comporte un lion :

(4) Donne-moi l'épée du lion.

16 De tels énoncés militent fortement contre l'hypothèse classique selon laquelle on doive déjà construire un sens littéral (que l'on rejette) pour ensuite comprendre le sens figuré. Wilson (2006) remarque d'ailleurs que le sens littéral de certains énoncés métaphoriques peut tout à fait correspondre à la réalité, sans que cela empêche de les comprendre métaphoriquement :

(5) L'ordre du jour de la réunion n'est pas gravé dans le marbre.

17 En ce sens, le mécanisme paraît proche de celui à l'œuvre avec les emplois approximatifs, où le sens littéral est étendu et compris comme une approximation (un type de modulation) :

(6) La Terre est ronde.

(7) L'Italie est une botte.

18 À proprement parler, la Terre n'est pas parfaitement ronde - puisqu'elle est aplatie aux pôles et qu'elle est couverte de chaînes de montagnes - et l'Italie n'est pas une botte, quoique sa forme puisse évoquer cet objet. Malgré tout, ces énoncés paraissent vrais car on reconstruit « ce qui est dit » comme une proposition modulée sans y penser, même si, 
en toute rigueur, il conviendrait plutôt d'attribuer à (6) et à (7) une valeur de vérité négative.

Les tropes ne relèveraient donc pas du sens implicite ou bien, si c'est le cas, pas au même titre que les implicatures. Plutôt que de tenter de définir la catégorie de l'implicite, nous nous cantonnons donc à noter la gageure de l'exercice et de simplement faire état, sans statuer, des mécanismes impliqués dans la dérivation du sens à partir des formes linguistiques.

\section{Principes d'inférence : problèmes théoriques}

Avant d'aborder les implicatures, qui tiennent une place d'honneur dans le sens implicite, un détour ou un retour au fondement théorique postulé pour leur genèse paraît plus que légitime. Les deux grands principes d'inférence, le principe de coopération et le principe de pertinence, présentent des angles d'approche différents qu'il convient de soumettre à l'analyse critique.

\subsection{Le principe de coopération}

21 Le modèle gricéen de dérivation des implicatures repose sur un principe de coopération se déclinant en quatre grandes catégories de maximes. Les écueils rencontrés par l'application du principe de coopération et des maximes conversationnelles ont été en grande partie reconnus par Grice lui-même (1989 : 368-372). Ils touchent en premier lieu au caractère arbitraire de l'architecture du modèle puisque, comme l'a toujours indiqué Grice (1989 : 26), les catégories de maximes suivent les catégories de Kant, et leur nombre précis est potentiellement sujet à débat et variation. Une des maximes de quantité, « $\mathrm{Ne}$ dites pas plus que ce qui est attendu de vous ", paraît ainsi faire double emploi avec la maxime de pertinence « Soyez pertinent ».

En second lieu, le principe de coopération n'est qu'une idéalisation, qui ne prévaut pas toujours. Grice (1989 : 369-370) remarque que, dans certaines conversations, des objectifs communs très ténus coexistent avec beaucoup de réserve, d'agressivité, de mauvaise foi, et que, dans d'autres où la coopération est simplement apparente (comme pour une interrogation contradictoire), le principe s'applique tout de même par défaut, parce que les participants font comme s'il l'était. Voici comment Grice introduit initialement l'idée de ce principe :

Nos échanges conversationnels... relèvent typiquement, ne serait-ce qu'à un certain degré, d'efforts de coopération; et chaque participant reconnaît à ceux-là, d'une manière ou d'une autre, un but commun ou un ensemble d'objectifs, ou tout du moins une direction mutuellement admise. (Grice 1989 : 26)

Grice postule que la coopération se trouve au cœur de nos échanges conversationnels, sous la forme d'un objectif partagé, ou d'une direction discursive commune, ce qui le conduit à la formulation de son principe de coopération :

un principe général assez imparfait que les participants sont censés (toutes choses égales par ailleurs) respecter, à savoir: que votre contribution conversationnelle soit telle qu'elle corresponde à ce qui est attendu de vous, au moment où elle intervient, en fonction du but ou de la direction de l'échange conversationnel dans lequel vous êtes engagé. (Grice 1989 : 26) 
an-Jacques Lecercle prend le contrepied de l'idée qu'un principe de coopération dirige nos interactions verbales. La confrontation parait tout aussi légitime pour fonder ces interactions, tant elle y est prégnante. Il donne pour exemple les conversations réelles, les dialogues des pièces de Pinter, les images de la guerre employés pour parler d'affrontements verbaux (1990: 247). Il va même jusqu'à avancer un principe de confrontation, qui pourrait régir les interactions verbales, prolongé par des maximes (1994: 72-100). La question de la dominance d'échanges coopératifs plutôt que conflictuels est en tout cas difficile à trancher, et c'est la vertu des travaux de Lecercle de le montrer. Il est toutefois raisonnable d'accorder que même les joutes verbales, comme les débats politiques, impliquent certaines règles en plus de tout un feuilleté d'objectifs pour les participants, dont certains peuvent être communs :

on ne peut concevoir d'échanges qui s'établissent sur un mode entièrement non coopératif : polémiquer, c'est encore partager, c'est (ad)mettre en commun un certain nombre de valeurs, et de règles du jeu linguistique et conversationnel. (Kerbrat-Orecchioni 1998 [1986] : 197)

Malgré les réserves et concessions consenties par Grice, sa conception rationnelle et irénique de la conversation apparaît tout de même singulièrement idéaliste, peu conforme à la réalité, et en tout cas non suffisamment fondée pour se traduire par un principe pragmatique général servant de moteur à tout un ensemble d'inférences.

\subsection{Le principe de pertinence}

Pour éviter de tels problèmes, aujourd'hui bien mis en évidence par les pragmaticiens qui s'intéressent aux phénomènes d'impolitesse et d'affront à la face d'autrui (Bousfield 2008, Culpeper 2011, parmi d'autres), Sperber et Wilson proposent la définition suivante de leur principe d'inférence, le principe communicationnel de pertinence :

Tout acte de communication ostensive communique la présomption de sa pertinence optimale.

(Sperber \& Wilson 1995 [1986], 158]

L'important est que ce principe s'applique sans exception (Sperber et Wilson 1995 : 167), car sinon il paraitrait difficile de faire valoir ce principe comme capable de fonder l'ensemble des inférences pragmatiques qui conduisent au sens. Naturellement, tout le monde n'est pas pertinent, mais quand un importun attire l'attention, il présume que ce qu'il dit est pertinent pour les autres, ce qui n'est en réalité pas souvent le cas. Il parait donc juste de parler d'une présomption de pertinence plutôt que d'une pertinence effective. Même des énoncés comme (8) sont susceptibles d'être interprétés comme garants d'une certaine pertinence, ne serait-ce que de façon détournée et hypocrite :

(8) Vous n'auriez pas un euro?

29

Celui qui vous demande cela dans la rue est potentiellement pertinent s'il part du principe que, disons, son allocutaire est quelqu'un de bon, de bien intentionné, soucieux des autres, et qu'il serait reconnaissant de savoir que quelqu'un comme le locuteur est dans le besoin. Dans le cas d'insultes directes qui ne se cachent pas, il parait difficile de postuler une application, même indirecte, du principe de pertinence. Dans la pièce Dommage qu'elle soit une putain (IV, iii) de John Ford, l'homme de main Vasques, qui s'apprête à torturer une suivante qu'il malmène déjà, lui lance, vraisemblablement en lui serrant le bâillon sur sa bouche :

(9) Je vais te travailler les gencives, salope au ventre de crapaud. 
L'insulte est en quelque sorte la continuation (voire le prélude) de la violence physique par un autre moyen, et force est de constater ici que la présomption de pertinence est vide. Il serait ici difficilement tenable de considérer que le principe est honoré ne seraitce que de façon hypocrite. Il n'est pas inapproprié de le rappeler ici sous forme condensée : «Souhaitant que son acte de communication soit effectif, le communicateur, du fait de cet acte même, indique sa volonté que son énoncé soit perçu comme pertinent, et c'est ce qu'affirme le Principe Communicationnel de Pertinence » (Wilson et Sperber 2012 : 6). Or en (9), toute prétention à la pertinence paraît abandonnée, cédant la place à la menace, à l'intimidation et à une agression psychologique opérée par le locuteur. On pourrait peut-être objecter que (9) est pertinent en ce que le locuteur souhaite attirer l'attention de son allocutaire sur le fait qu'il est utile pour elle de savoir qu'il le méprise, par l'intermédiaire de l'acte ostensif qu'est sa prise de parole. Mais ce n'est pas là une description adéquate de ce qui se passe. Le locuteur cherche simplement à rabaisser (à provoquer, à réduire, à blesser) l'allocutaire.

31 Nous proposons une expérience de pensée pour étayer la démonstration : en raisonnant par l'absurde, si le principe de pertinence s'appliquait à cet énoncé, il faudrait alors considérer que donner un coup de pied ou lancer une pierre à quelqu'un, actes ostensifs par excellence, serait un acte de communication en ce qu'il serait pertinent pour la cible de savoir qu'on lui veut du mal. Pourquoi refuser aux gestes un statut communicatif alors qu'on l'accorderait à l'énoncé (9) ? Avancer que la parole déclencherait automatiquement l'application du principe n'est pas satisfaisant car le principe lui-même ne serait plus justifié, parce qu'infondé dans les faits, alors même que le principe a pour but de fournir un fondement pour inférer l'implicite des énoncés et des comportements ostensifs visant à la communication. Autant dire que cela conduit à la circularité. Toujours est-il qu'un principe demeure a priori nécessaire afin de déclencher les inférences pragmatiques. Déduire de cette nécessité la validité de tel principe ou de tel autre est une ouverture théorique à exclure. La question reste donc entière : sur quel principe ou bases fonder les inférences pragmatiques?

\section{Deux modèles de dérivation du sens}

C'est à la question du fondement des inférences pragmatiques que la dernière partie de notre contribution va s'attacher à répondre, ne serait-ce que de manière exploratoire, à partir de la comparaison de deux modèles de dérivation du sens: la théorie de la pertinence et celle de Recanati. Une autre approche sera ébauchée, postulant qu'une présomption de pertinence ou de coopération n'est utile que pour lancer des inférences dans deux cas bien précis.

\subsection{Théorie de la pertinence : explicatures et implicatures}

En théorie de la pertinence, le principe de pertinence s'applique pour dériver à la fois « ce qui est dit ", ou plus exactement les explicatures dans ce cadre théorique, et donc pas uniquement les implicatures. Voici comment se définit une explicature :

explicature : une assomption communiquée de manière ostensive qui est dérivée par inférence à partir d'une des représentations incomplètes (formes logiques) encodées par l'énoncé.

(Carston $2002: 377$ ) 
Une explicature correspond à une proposition que l'on développe à partir des représentations logiques incomplètes fournies par un énoncé. Illustrons à partir de (10) :

(10) Je n'ai plus de liquide, je vais passer à la banque.

En plus de l'attribution de la référence de «je» au locuteur, il convient en outre de désambiguïser liquide, et d'enrichir pragmatiquement la dénotation de banque, car il s'agit en toute vraisemblance d'un distributeur automatique de billets que le locuteur a à l'esprit (emploi vague du mot, ou bien spécialisé: 'l'institution banque telle qu'elle se manifeste par l'intermédiaire du distributeur'). Une explicature adéquate peut ainsi être obtenue, à travers le jeu d'inférences guidées par le principe de pertinence, qui garantit un rapport optimal (suivant les capacités et les préférences du locuteur) entre pertinence du contenu et efforts requis pour l'interprétation. Cela revient à dire que l'on suit une procédure heuristique de compréhension qui consiste à suivre une interprétation demandant le moindre effort, jusqu'à ce que soit satisfaite l'attente de pertinence de la part de l'allocutaire (Sperber, Cara et Girotto 1995 : 51, Wilson et Sperber $2012: 6-7$ ). En pratique ici, liquide se comprend donc comme une forme d'argent, banque prend le sens le plus naturel dans ce contexte, et on suppose que le locuteur parle de lui-même en utilisant je, plutôt que de donner dans une citation de quelqu'un d'autre à la première personne. Suivant le même guidage heuristique, l'allocutaire serait à même de reconstruire l'implicature 'Je vais retirer des billets au distributeur'.

Pour dériver des implicatures, Sperber et Wilson (1995 : 93-103) postulent l'existence d'un appareil déductif, capable de dériver des implicatures correspondant à des prémisses ou à des conclusions de syllogismes que l'on peut reconstruire. Pour reprendre un exemple classique de Grice :

(11) A : Je n'ai plus d'essence.

$\mathrm{B}$ : Il y a un garage au coin de la rue.

On peut reconstruire pour (11) le syllogisme suivant :

Mineure : Il y a un garage au coin de la rue.

Majeure : Dans un garage, on trouve de l'essence.

Conclusion : Dans le garage au coin de la rue, on trouve de l'essence. entre essence et garage et correspond à une connaissance encyclopédique partagée (à l'époque où Grice écrivait, les garages vendaient de l'essence). Le syllogisme permettant de comprendre que le locuteur de (10) va retirer de l'argent au distributeur est bien plus banal :

Mineure : le locuteur a besoin d'argent liquide.

Majeure: Quand on a besoin d'argent liquide, on va retirer des billets au distributeur.

Conclusion : Le locuteur va retirer des billets au distributeur.

La majeure est une nouvelle fois évoquée par l'activation des concepts d'argent liquide et de distributeur, dont il est question dans l'énoncé (10). D'autre part, il est également légitime de considérer comme relevant du savoir collectif que lorsqu'on n'a plus d'argent liquide (ce qui est dit dans l'énoncé concernant le locuteur), on a besoin d'en avoir. Nous voyons de nouveau comment l'appareil déductif fonctionne de concert avec le principe de pertinence pour générer l'implicature voulue. 


\subsection{Après Grice : le modèle de Recanati}

Kent Bach (2006: 24-28) estime que Grice lui-même ne cherchait pas nécessairement à limiter l'application de son principe de coopération et de ses maximes à la dérivation des implicatures, et il n'est pas exclu pour lui d'invoquer ces derniers pour déterminer «ce qui est dit» et inférer ce qu'il appelle des implicitures (correspondant chez lui au comblage et à l'expansion : voir 1.2 et la note 2). Recanati (2004:21) propose quant à lui le modèle suivant, stipulant que « ce qui est dit » demeure accessible aux participants d'une conversation :

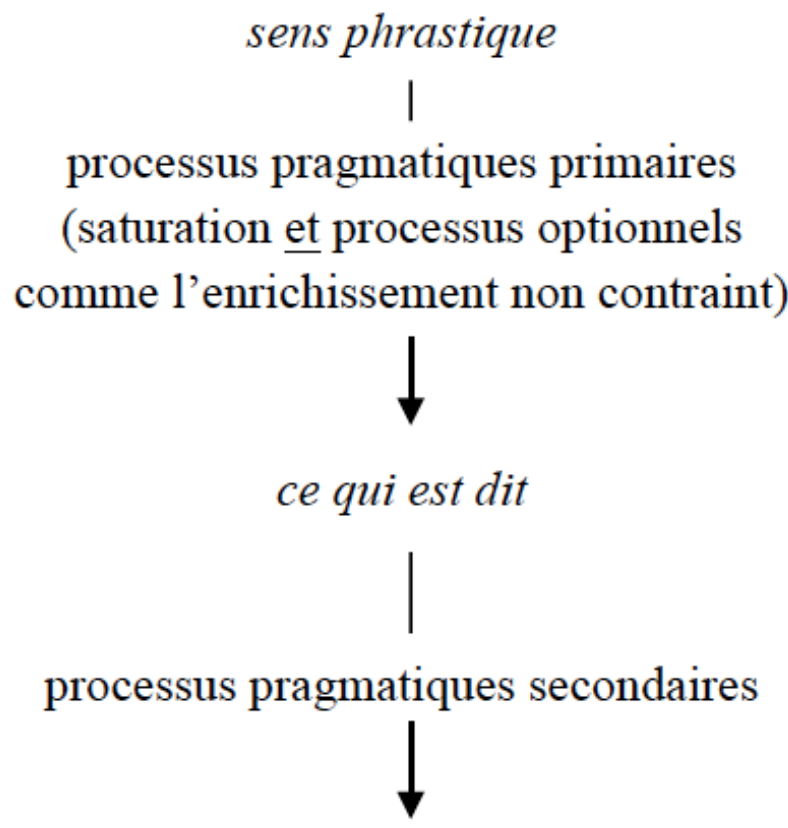

ce qui est communiqué

41 Selon Recanati (tout comme, d'ailleurs, selon Sperber et Wilson et la lecture que Bach fait de Grice), les implicatures peuvent commencer à être inférées sans que "ce qui est dit » (ou les explicatures) soit définitivement fixé. Le schéma ci-dessus ne doit pas être lu comme ayant des implications chronologiques sur la construction du sens.

L'originalité du modèle de Recanati réside dans le fait que les processus pragmatiques primaires ne font pas appel à un principe de communication quelconque, mais ils opèrent de manière automatique et associative, contrairement aux processus pragmatiques secondaires qui, eux, fonctionnent suivant le modèle gricéen classique de communication.

Dans le modèle que j'ai à l'esprit, le sens littéral d'une expression est celui auquel on a tout d'abord accès, et c'est lui qui déclenche l'activation de représentations qui y sont associées. Ce sens littéral est un candidat naturel pour recevoir le statut de valeur sémantique, mais il y en a d'autres : certaines représentations activées par association fournissent d'autres candidats au statut de valeur sémantique. (Recanati $2004: 28)$

Dit autrement, ce qui est dit correspond au sens qui est sélectionné en fonction du contexte. Nul besoin d'évoquer un principe particulier pour comprendre ce qui est dit. 
Cette façon d'envisager la genèse du sens n'est pas sans évoquer les modèles neuronaux, qui fonctionnent également suivant des principes d'activation et d'association (entre autres) $)^{5}$. Dans l'exemple (10), il nous semble raisonnable de considérer que je active une représentation du locuteur (à moins d'une inhibition du processus), tandis que les sens pertinents de liquide et de banque apparaissent à la suite de l'activation du réseau neuronal (dans le sens donné à neural web par Pulvermüller 2002) correspondant au sens voulu, les autres sens et réseaux correspondants étant finalement écartés par le contexte et leur activation inhibée. La question est de savoir si ce traitement peut s'appliquer à l'ensemble des algorithmes qui conduisent à « ce qui est dit » ou non. ${ }^{6}$ Toujours est-il que pour certains des processus en question, il est envisageable de proposer des dérivations sans nécessairement invoquer un principe de communication et d'inférence. C'est un résultat souhaitable si l'on pense que les principes généraux qui ont été proposés jusqu'ici sont (au moins en partie) inadéquats.

\subsection{De l'utilité relative des principes et de la présomption qu'ils véhiculent}

Contrairement à Recanati, nous n'acceptons pas le cadre théorique (néo-)gricéen et nous recourons à l'appareil déductif de Sperber et Wilson pour dériver les implicatures, comme pour l'énoncé (11). Il nous reste à savoir si à lui seul, cet appareil est adéquat afin de dériver toutes les implicatures potentiellement communiquées sans recourir à un principe d'inférence général. On pourrait ainsi imaginer qu'un traitement identique ou similaire s'applique aux processus pragmatiques primaires et secondaires, comme le suggère nos analyses de (10) et de (11).

Il demeure néanmoins nécessaire de postuler que, par défaut, une présomption ou un principe général de communication est respecté dans deux grands cas. En premier lieu, le recours à un postulat de pertinence ou de coopération est idoine pour justifier le surcoût interprétatif que demande un énoncé difficile ou obscur, lorsque l'allocutaire doit consentir à fournir un effort conscient, auquel l'invite le postulat. C'est notamment le cas de styles ou de métaphores qui ne sont pas tout de suite limpides (comme pour la métaphore présentée hors contexte dans l'énoncé isolé Sally est une fine pluie de printemps, emprunté à Jean-Jacques Lecercle). L'allocutaire doit alors guider la dérivation du sens en avançant des hypothèses sur ce sens, en les vérifiant, quittant le mode d'interprétation automatique pour suivre une procédure plus lente, plus complexe, qui fait intervenir la réflexion consciente. L'allocutaire peut alors naturellement décider de ne pas fournir l'effort interprétatif qu'on lui demande, le refuser, et cela même si le locuteur tend visiblement à signifier qu'interpréter son énoncé apportera un certain bénéfice (informatif et/ou cognitif) pour celui à qui il s'adresse. Un étudiant rétif à la poésie d'un représentant du mouvement symboliste illustre bien ce choix. Si le locuteur paraît ne pas respecter cet usage plus ou moins attendu dans une interaction verbale qui, après tout, en tant qu'activité humaine au sein d'une société, tendrait plutôt en moyenne vers une certaine harmonie (quoique ce ne soit pas toujours le cas), l'allocutaire n'a pas de motivation directe pour interpréter l'énoncé.

En deuxième lieu, un postulat de coopération, de bonne volonté communicationnelle, ou une présomption de pertinence optimale est requise de sorte à pouvoir dériver certaines assomptions ${ }^{7}$. Considérons (12), un exemple emprunté à Recanati (2010:15) :

(12) Il s'est cassé un doigt.

Corela, HS-25 | 2018 
Des enoncés tels que (12) nécessitent le postulat d'une présomption de pertinence (ou d'une présomption équivalente) en vue de reconstruire les syllogismes permettant de dériver les assomptions voulues. À partir de Il s'est cassé un doigt, on comprend que si un accident plus grave s'était produit (un doigt et trois côtes cassés, tout le bras fracturé, etc.), le locuteur l'aurait dit, et que, donc, il n'a rien de plus grave. Voici le raisonnement :

Mineure : Le locuteur dit qu'une tierce personne s'est cassé un doigt.

Majeure : Si on décrit un résultat pour un accident, on ne cache pas l'étendue de celui-ci.

Conclusion : La personne en question a un doigt cassé, et rien de plus.

La majeure du syllogisme ne peut s'obtenir à partir du contexte seul: c'est une application en situation d'une présomption de pertinence ou de bonne volonté communicationnelle (qui correspond en contexte à la mise en pratique d'une maxime de quantité, ou bien de la loi d'exhaustivité de Ducrot 1991: 134). Le fait de parler d'un accident crée l'attente d'un résultat tragique (ou simplement mauvais) et, dans l'optique d'une coopération ou d'une pertinence visée, le locuteur se doit de dire le pire. Nous avons proposé dans un autre article (2013: 33) qu'un tel postulat, même s'il ne correspond qu'à une idéalisation, peut se considérer comme une hypothèse employée par défaut par les allocutaires, qui serait fossilisée en quelque sorte, en vertu de sa vérification incertaine mais routinière dans les interactions verbales. Cela ne revient toutefois pas à dire qu'une telle hypothèse est vraie pour tous les énoncés qui peuvent être produits.

\section{Conclusion}

Caractériser l'implicite n'est pas chose aisée, puisque des processus pragmatiques inférentiels sont déjà sollicités pour déterminer «ce qui est dit », le sens propositionnel qui correspond aux énoncés, modulé tandis qu'il est reconstruit par les allocutaires, et qui demande déjà le traitement de tropes comme la métaphore pour attribuer une référence à certains arguments logiques. Les principes d'inférence postulés dans les écrits de Grice et certains travaux qu'ils ont inspirés, le principe de coopération (avec les maximes qui lui sont liées) tout autant que le principe communicationnel de pertinence, présupposent une vision trop idéaliste et idéalisée des interactions verbales, qui sont au moins sporadiquement marquées par l'intimidation et la manifestation de violence psychologique ou symbolique sans que leurs auteurs ne dissimulent leurs intentions de façon hypocrite - ce qui pourrait d'ailleurs nuire à l'efficacité de leur agression verbale. Ces principes ne sont donc pas à même de fonder l'ensemble des inférences pragmatiques si on les suppose sans exception. Il n'en reste pas moins qu'une présomption de pertinence ou de coopération constitue une hypothèse qui, bien que non toujours vraie, est utile afin de rendre compte de deux grands cas de figure: le surcoût d'effort interprétatif conscient que certains énoncés demandent et que cette hypothèse encourage à fournir (sans garantie que cet effort soit consenti) et, d'autre part, la dérivation d'une classe d'assomptions par le biais de l'appareil déductif, nécessitant la reconstruction d'un syllogisme pour lequel l'hypothèse en question est mobilisée par défaut afin d'établir le contenu d'une des prémisses (la majeure). En outre, «ce qui est dit» peut être au moins en partie obtenue par un simple jeu d'activations et d'associations, guidées par le contexte, suivant le modèle des réseaux neuronaux et la 
suggestion de Recanati. Cependant, contrairement à lui, nous n'acceptons pas le cadre théorique (néo-)gricéen, et il nous paraît nécessaire de recourir à l'appareil déductif de Sperber et Wilson, pleinement sollicité pour dériver les implicatures.

\section{BIBLIOGRAPHIE}

BACH K. (1994a), “Semantic slack”, in TSOHATZIDIS S. (éd.) Foundations of Speech Act Theory, Londres, Routledge, p. 267-291.

BACH K. (1994b), “Conversational impliciture”, Mind and Language, n 9, p. 124-162.

BACH K. (2006), "The top 10 misconceptions about implicature”, in BIRNER B-J. et WARD G. (éds) Drawing the Boundaries of Meaning, Amsterdam, John Benjamins, p. 21-30.

BOUSFIELD D. (2008), Impoliteness in Interaction, Amsterdam, John Benjamins.

CARSTON R. (2002), Thoughts and Utterances: The Pragmatics of Explicit Communication, Oxford, Blackwell.

CULPEPER J. (2011), Impoliteness: Using Language to Cause Offence, Cambridge, Cambridge University Press.

DUCROT O. (1969), « Présupposés et sous-entendus », Langue Française, n 4, p. 30-43.

DUCROT O. (1991), Dire et ne pas dire, $5^{\mathrm{e}}$ edition, Paris, Hermann.

GRICE H-P. (1975), "Logic and conversation”, dans COLE P. \& MORGAN J. (éds), Syntax and Semantics, vol. 3, New York, Academic Press, p. 41-58 (Reproduit dans Grice 1987, p. 22-40).

GRICE H-P. (1989), Studies in the Ways of Words, Harvard, Harvard University Press.

KERBRAT-ORECCHIONI C. (1998 [1986]), L'implicite. $2^{\mathrm{e}}$ édition, Paris, Armand Colin.

LARREYA P. (2008), « Utilisations stylistiques des formes présuppositionnelles », Bulletin de la Société de Stylistique Anglaise, n 30, p. 225-236.

LECERCLE J-J. (1990), The Violence of Language, Londres, Routledge.

LECERCLE J-J. (1994), Philosophy of Nonsense, Londres, Routledge.

MOESCHLER J. (1981), « Discours polémique, réfutation et résolution des séquences conversationnelles ", Cahiers de Linguistique française, $\mathrm{n}^{\circ}$ 1, p. 54-78.

NOVECK I-A. \& SPERBER D. (2007), “The why and how of experimental pragmatics: The case of 'scalar inferences"', in BURTON-ROBERTS N. (éd.), Advances in Pragmatics, Basingstoke, Palgrave, p. 181-212 (Reproduit dans D. Wilson et D. Sperber 2012, p. 307-330).

PULVERMÜLLER F. (2002), The Neuroscience of Language, Cambridge, Cambridge University Press.

RECANATI F. (2004), Literal Meaning, Cambridge, Cambridge University Press.

RECANATI F. (2010), Truth-Conditional Pragmatics, Oxford, Oxford University Press. 
SIMONIN O. (2010), «Impolitesse, coopération et principes d'inférence », Lexis special, $\mathrm{n}^{\circ} 2$ : impolitesse/impoliteness, p. 21-34 : http://lexis.univ-lyon3.fr/IMG/pdf/Lexis_special_2__Simonin.pdf (revue électronique, dernièrement consultée le 25/02/2016)

SIMONIN O. (2013), « Typologie des contenus implicites », Études de Stylistique Anglaise, $\mathrm{n}$ $\circ$, p. 27-42.

SPERBER D., CARA F. \& GIROTTO V. (1995), "Relevance theory explains the selection task", Cognition, $\mathrm{n}^{\circ}$ 57, p. 31-95.

SPERBER D. \& WILSON D. (1995 [1986]), Relevance: Communication and Cognition, Oxford, Blackwell. SPERBER D. \& WILSON D. (2004), "Relevance theory”, in HORN L-R. \& WARD G. (éds) The Handbook of Pragmatics, Oxford, Blackwell, p. 607-632.

WILSON D. (2006), “The pragmatics of verbal irony: echo or pretence?”, Lingua, $\mathrm{n}^{\circ} 116$, p. 1722-1743.

WILSON D. \& SPERBER D. (2012), Meaning and Relevance, Cambridge, Cambridge University Press.

\section{NOTES}

1. L'un des corollaires, suivant lequel un contenu imposé est implicite, se retrouve encore fréquemment dans les écrits de linguistes, comme sous la plume de Larreya (2008) : l'idée que l'on peut avoir un contenu explicite qui véhicule un présupposé, comme ce serait le cas pour (1), paraît contradictoire, bien que le simple fait de nommer un référent permette effectivement parfois d'imposer un contenu à son allocutaire (voir Simonin 2013 : 37-39). Catherine KerbratOrecchioni se montre prudente pour ce cas de figure, indiquant dans une note se rapportant à (1) que «le statut de présupposé des informations liées à l'adjectif épithète [aveugle] ... n'est pas unanimement admis » (Kerbrat-Orecchioni $1998: 356)$.

2. Les deux premières catégories viennent de Grice, le terme comblage (completion) de Kent Bach, saturation (repris en théorie de la pertinence, notamment par Carston) et modulation de Recanati (qui correspond, mutatis mutandis, à l'expansion de Kent Bach). La modulation inclut des processus comme l'extension (métonymique), le transfert (métaphorique), l'approximation (les emplois approximatifs de syntagmes comme de l'adjectif ronde dans La Terre est ronde) et la restriction lexicale (J'ai apporté une bonne bouteille implique généralement qu'il s'agit d'une bouteille de vin ou d'un autre alcool). De la modulation relève l'enrichissement pragmatique non contraint (free enrichment) de Sperber et Wilson, c'est-à-dire d'un enrichissement qui n'est pas simplement déclenché par l'emploi de formes linguistiques, et qui s'oppose à la restriction lexicale. Robyn Carston (2002: 28) propose six mécanismes, développant l'ambiguïté sous les perspectives du sens notionnel et de la portée d'opérateurs, et la modulation sous celles de l'approximation et de la restriction lexicales.

3. Cette position s'inscrit en porte-à-faux par rapport au minimalisme sémantique, que critique abondamment Recanati (2004, 2010).

4. La traduction de l'anglais nous est due, comme les autres qui figurent dans cette contribution.

5. On retrouve également là une des fonctions du principe cognitif de pertinence (par opposition au principe communicationnel) stipulant que la cognition humaine vise à la maximisation de pertinence: «les mécanismes de recherche des humains tendent spontanément à activer des assomptions potentiellement pertinentes » (Wilson et Sperber 2012:6)

6. Le fait que les liens métaphoriques soient profondément ancrés dans notre cognition va dans ce sens, et suggère que des modulations comme le transfert métaphorique peuvent se prêter au même traitement. L'activation d'un concept correspondant à un sens métaphorique, figuratif, 
conduit bien souvent à favoriser l'activation du concept non figuratif qui lui est associé. Ainsi, un homme de pouvoir paraîtra plus grand qu'il l'est réellement à qui le rencontre, et un sujet à qui l'on demande de se remémorer le pire acte qu'il ait commis le conduit littéralement à voir le monde comme plus sombre et à être corrélativement attiré par des sources de lumière (Cerveau et Psycho 51, 2012: 8).

7. Nous empruntons ce terme à Sperber et Wilson pour ne pas préjuger du statut de ces assomptions, qui peuvent refléter «ce qui est dit» (ce sont les explicatures de la théorie de la pertinence) ou bien être des implicatures.

\section{RÉSUMÉS}

Cet article traite du lien entre l'implicite et les grands principes d'inférence (coopération, pertinence). L'implicite est une catégorie étendue qu'il n'est pas facile de circonscrire. Un des acquis de la pragmatique post-gricéenne est que les inférences entrent déjà en ligne de compte pour dériver "ce qui est dit » ou les explicatures, repoussant ainsi les frontières de la notion d'implicite. Nous montrons que les principes d'inférence postulés ne sont pas à l'abri d'être contredits dans les faits (comme dans le cas d'agression verbale frontale), compromettant ainsi leur validité. Cependant, il est en partie possible de ne pas y recourir si, à la suite de Recanati (2004), on considère qu'un modèle parallèle, associatif, est à même de conduire à la reconstruction de "ce qui est dit ». Il demeure toutefois nécessaire de les invoquer de façon minimale pour dériver une classe spécifique d'assomptions présentée ici, tout comme pour rendre compte du fait qu'une présomption générale de coopération ou de pertinence semble être émise par défaut (sans signe du contraire), invitant les allocutaires à fournir un certain effort interprétatif lorsque le sens d'un énoncé n'apparaît pas tout de suite clairement.

This paper explores the connection between general pragmatic principles (Cooperation, Relevance). Implicit content is a broad category. Post-Gricean pragmatics has consistently shown that inferences already come into play in order to derive " what is said », or explicatures, rolling back the boundaries of the notion of implicit meaning. I argue that the general pragmatic principles postulated are not unlikely to be honoured in the breach (as in cases of frontal verbal aggression), which compromises their validity. However they can be partly dispensed with if, following Recanati (2004), we assume that an associative, parallel model of processing is sufficient to reconstruct " what is said ». Yet they still need to be invoked minimally to derive a specific class of assumptions presented here, as well as to account for the fact that some general presumption of cooperation or relevance appears to be made by default (with no evidence to the contrary), inviting hearers to provide a modicum of interpretative effort when the meaning of an utterance is not immediately clear.

\section{INDEX}

Mots-clés : implicature, explicature, « ce qui est dit », principe de pertinence, principe de coopération

Keywords : implicature, explicature, "what is said", principle of relevance, cooperative principle 
AUTEUR

OLIVIER SIMONIN

CRESEM, EA 7397, Université de Perpignan-Via Domitia 